\title{
Chronic cerebrospinal venous insufficiency in multiple sclerosis: a highly prevalent age-dependent phenomenon
}

\author{
Roberta Lanzillo ${ }^{1,5^{\dagger}}$, Marcello Mancini ${ }^{2,3 \dagger}$, Raffaele Liuzzi ${ }^{2,4^{*}+}$, Orlando Di Donato ${ }^{4}$, Elena Salvatore ${ }^{4}$, \\ Valentina Maglio ${ }^{4}$, Giovanni Vacca ${ }^{4}$, Luca Amato ${ }^{1}$, Gennaro D'Anna ${ }^{4}$, Arturo Brunetti ${ }^{4}$ and Vincenzo Brescia Morra ${ }^{1}$
}

\begin{abstract}
Background: This study aimed to investigate the prevalence and clinical relevance of chronic cerebrospinal venous insufficiency (CCSVI) in multiple sclerosis (MS) patients and healthy controls using extra- and intracranial colour Doppler sonography.

Methods: We examined 146 MS patients, presenting with a clinically isolated syndrome, relapsing-remitting, secondary progressive, or primary progressive MS, and 38 healthy controls. Sonographic examination was performed according to Zamboni's protocol and was performed by three independent sonographers. The results of sonographic examination were compared with clinical and demographic characteristics of the patients.

Results: CCSVI, defined as the presence of at least two positive Zamboni's criteria, was found in $76 \%$ of MS patients and $16 \%$ of control subjects. B-mode anomalies of internal jugular veins, such as stenosis, malformed valves, annuli, and septa were the most common lesions detected in MS patients (80.8\%) and controls (47.4\%). We observed a positive correlation between sonographic diagnosis of CCSVI and the patients' age $(p=0.003)$. However, such a correlation was not found in controls $(p=0.635)$. Notably, no significant correlations were found between sonographic signs of CCSVI and clinical characteristics of MS, except for absent flow in the jugular veins, which was found more often in primary $(p<0.005)$ and secondary $(p<0.05)$ progressive patients compared with non-progressive patients. Absent flow in jugular veins was significantly correlated with patients' age ( $p<0.0001)$.
\end{abstract}

Conclusions: Sonographically defined CCSVI is common in MS patients. However, CCSVI appears to be primarily associated with the patient's age, and poorly correlated with the clinical course of the disease.

Keywords: Multiple sclerosis, Venous insufficiency, Ultrasonography

\section{Background}

Multiple sclerosis (MS) is an autoimmune disease of the central nervous system (CNS) characterized by inflammation, demyelination, and neurodegeneration [1]. However, neuropathological studies have underlined the presence of a subset of patients with a pattern that closely mimics tissue alterations found in the early stages of white matter ischemia [2]. Moreover, cerebral perfusion studies have

\footnotetext{
*Correspondence: raffaele.liuzzi@cnr.it

${ }^{\dagger}$ Equal contributors

${ }^{2}$ Institute of Biostructure and Bioimaging, National Research Council of Italy (CNR), Naples, Italy

${ }^{4}$ Department of Biomorphological and Functional Science, Federico II University School of Medicine, Naples, Italy

Full list of author information is available at the end of the article
}

shown diffuse hypoperfusion in patients with MS compared with age-matched controls with a greater cerebral blood flow decrease in primary progressive (PP) MS compared with relapsing-remitting (RR) MS [3]. Recently, a strong correlation between MS and a vascular condition called chronic cerebrospinal venous insufficiency was reported [4].

Chronic cerebrospinal venous insufficiency (CCSVI) is characterized by anomalies of the main extracranial cerebrospinal venous routes. CCSVI is detected by selective venography [4-10] and extracranial venous echo-colour Doppler (ECD) [4,9-12] with higher sensitivity and specificity than magnetic resonance venography (MRV) [9]. The prevalence of CCSVI in MS patients is highly variable

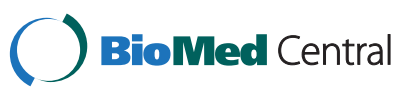


$[4,10,11,13]$. The largest study conducted to determine the prevalence of CCSVI in patients with MS, those with clinically isolated syndrome (CIS), those with other neurological diseases, and healthy controls (HCs), using echocolor Doppler (ECD), showed an increased prevalence of CCSVI in MS [14]. This finding suggests that CCSVI does not have a primary causative role in the development of MS. However, perfusion studies have shown a decrease in cerebral perfusion in MS subjects and HCs with CCSVI $[15,16]$.

To date, there are only few studies regarding the relationship between venous abnormalities, phenotype, and the clinical course of MS [17-19], with different and sometimes conflicting results.

This study aimed to investigate the prevalence of CCSVI in a representative MS population and HCs using ECD. We also investigated the clinical relevance and correlations of CCSVI with MS disease parameters.

\section{Methods \\ Patients}

This single-centre, cross-sectional study included 171 consecutive MS patients and 41 sex- and age-matched HCs. Inclusion criteria were diagnosis of MS, according to the McDonald criteria [20], including RR, secondary progressive (SP) MS, and PPMS, as defined by Lublin [21], and diagnosis of CIS. The HC group included relatives of MS patients and healthy volunteers. For all patients, exclusion criteria were the presence of relapse and steroid treatment in the 30 days preceding study entry and, for HCs, a history of cerebral congenital vascular malformations and pre-existing medical conditions known to be associated with brain pathology (e.g., cerebrovascular disease and a positive history of alcohol abuse).

\section{Study assessments}

All of the participants underwent a clinical examination and intra- and extracranial ECD of the neck in the same week. Biochemistry was performed to exclude haematological or other medical conditions that could affect haemorheology. Standard demographic and clinical information on all participating subjects were acquired. This included, but was not limited to age, sex, familial history, detailed medical history of vascular risks with particular emphasis on venous diseases, age at disease onset, age at diagnosis, time interval between first and second relapses, annualized relapse rate, and current and previous therapy information. Disease onset symptoms were retrospectively recorded and classified into five categories according to the Expanded Disability Status Scale (EDSS) functional system involved: visual, pyramidal, sensory, subtentorial (cerebellar and brain-stem), and spinal symptoms. Actual disease phenotype was also assessed and divided into the same five categories, according to the most disabled functional system at the time of evaluation. A physical examination was performed with measurement of blood pressure, and EDSS [22] and single EDSS functional systems scores, and Multiple Sclerosis Severity Scale (MSSS) [23] scores were calculated, and disease subtype was classified.

\section{Standard protocol approvals, registrations, and patient consents}

This study was approved by the "Carlo Romano" ethics committee of the Federico II University of Naples, and informed consent was obtained from all subjects.

\section{Colour Doppler sonography evaluation}

Colour Doppler sonography was performed by the same radiologist (MM with greater than 20 years of vascular ultrasound experience) with the iU22 Ultrasound System (Philips, Amsterdam, The Netherlands) equipped with a 3.0-9.0 MHz linear wide-band transducer, a 5.0-8.0 MHz microconvex probe, and a $1.0-5.0 \mathrm{MHz}$ phased array transcranial probe.

Intracranial and extracranial venous outflow were evaluated according to the Zamboni criteria, based on the detection of five criteria as previously described [4]. A subject was considered CCSVI-positive if two or more Zamboni criteria were fulfilled. The radiologist that performed ECD was not blinded to the disease status of patients.

The images were stored and anonymously coded with an identification number. A further evaluation was performed off-line and independently repeated twice by two blinded radiologists (OD, VM) who reviewed all the images. The CCSVI diagnosis was considered valid only if the findings of the three radiologists agreed for each single criterion.

\section{Statistical analysis}

Continuous data are reported as mean and standard deviation or median and range, and categorical data as percentages. For descriptive statistics and estimates of prevalence, the $t$-test, the $U$ test (Mann-Whitney), Fisher's exact test, and the $X^{2}$ test were used. Despite the fact that diagnosis of MS is not a gold standard test to diagnose CCSVI, sensitivity, specificity, and relative odds ratios (ORs) between HCs and patients with MS were calculated, using direct computation from $2 \times 2$ tables, to obtain results easily comparable with previous studies [4,14]. Prevalence rates for each of the five Zamboni criteria, as well as for different CCSVI presence groups, were calculated. Residual analysis was performed to assess differences in the distribution of these venous criteria among MS subtypes. Logistic regression techniques were used to evaluate correlations between CCSVI presence and clinical parameters. Significance was denoted when $\mathrm{p}$ was $<0.05$ 
by using two-tailed tests. Statistical analyses were performed using SPSS (version 18.0 Chicago, IL).

\section{Results}

Demographic and clinical data of MS patients and control groups are shown in Table 1. Significant differences in age, EDSS, and disease duration were evident in the subgroups. MS patients belonged to four clinical subtypes (53 RR, 58 SP, 19 PP, and 16 CIS), and were treated in 70\% of cases with various therapies (59 with interferon beta-1, 30 with natalizumab, 8 with glatiramer acetate, 3 with fingolimod, 1 with azathioprine, and 1 with mitoxantrone). Of the remaining 44 untreated patients, eight were naive to any disease modifying therapy, while the others had been previously treated with one or more different drugs.

It was not possible to assess all 5 Zamboni criteria for the absence of an adequate temporal window or for image artefacts that had not allowed full exploration of the jugular valve plane in nine patients $(5 \%)$. Concordance among the three ultrasonographers on the presence or absence of CCSVI was found in 146/171 (85.4\%) MS patients and $38 / 41(92.7 \%)$ HCs who were included in the statistical analysis.

One hundred and eleven MS patients (76\%) and six HCs (16\%) were considered as CCSVI-positive (Figure 1, $\mathrm{p}<0.001)$. The sensitivity and specificity of the presence of CCSVI for MS were 76\% (95\% CI 68.6-82.4) and 84\% (95\% CI 78.3-93.1), respectively, with an OR of 16.9 (95\% CI 6.5-43.8) (Table 2). The prevalence of one or more positive Zamboni criteria was higher in the MS group than in the $\mathrm{HC}$ group $(93.2 \%$ vs $63.2 \%, \mathrm{p}<0.001)$. For three fulfilled criteria, the prevalence was significantly higher in the MS group (32.2\%) than in the HC group (7.9\%, $\mathrm{p}<0.003)$.
The prevalence of five single venous criteria is shown in Figure 1. B-mode abnormalities of the internal jugular vein (IJV) presenting as stenosis, malformed valves, annulus, and septa (criterion 3), were the most frequently detected anomalies in MS patients (80.8\%) and HCs (47.4\%), followed by reflux in the jugular or vertebral vein (criterion 1) (63.0\% in MS and $21.1 \%$ in $\mathrm{HCs}$ ).

CCSVI was equally distributed in the different MS subtypes $\left(\chi^{2}=0.583, \mathrm{p}=0.90\right)$, while the absence of detectable flow in the IJV or in the vertebral vein (VV) (criterion 4) was significantly less frequent in the nonprogressive forms compared with the progressive forms of the disease $(11.3 \%$ in RR and $6.3 \%$ in CIS patients, vs $57.9 \%$ in $\mathrm{PP}, \mathrm{p}<0.005$; and vs $36.2 \%$ in $\mathrm{SP}, \mathrm{p}<0.05)$ (Figure 1). The presence of CCSVI was positively related to age in the whole sample $(\mathrm{p}=0.001)$ and in the MS group ( $\mathrm{p}=0.003)$, but not in HCs $(\mathrm{p}=0.635)$. No correlations were found between the presence of CCSVI and sex or clinical parameters, such as age at onset, EDSS, MSSS score, disease duration, number of total relapses, relapses in the last 2 years, and mostly impaired EDSS functional system. Onset symptoms were found in 132 patients $(90.4 \%)$. No difference in the prevalence of CCSVI was found in relation to disease onset symptoms. Therapy parameters (number of subsequent diseasemodifying therapies to which patients had been exposed and the total time of therapy exposure) were not related to the presence of CCSVI.

Single venous criterion 1,3 , and 4 positivity was related to age in SM patients $(\mathrm{p}=0.029,0.012$, and 0.0001 , respectively). In particular, criterion 1 was strongly related to age in $R R$ patients $(p=0.006)$ and criteria 4 in $P P$ patients $(\mathrm{p}=0.01)$

Table 1 Demographic and clinical characteristics of HCs, all MS patients, and MS subtypes

\begin{tabular}{|c|c|c|c|c|c|c|c|c|}
\hline & \multicolumn{8}{|c|}{ Disease group (n) } \\
\hline & HCs (38) & All MS (146) & $P$ value & CIS (16) & PPMS (19) & RRMS (53) & SPMS (58) & $P$ value \\
\hline Age (y) & $37 \pm 10$ & $40 \pm 9$ & 0.112 & $32 \pm 7$ & $48 \pm 11$ & $37 \pm 9$ & $43 \pm 7$ & $0.001^{a}$ \\
\hline \multicolumn{9}{|l|}{ mean $( \pm \mathrm{SD})$} \\
\hline$M / F$ & $18 / 20$ & $49 / 97$ & 0.115 & $6 / 10$ & $6 / 13$ & $13 / 40$ & $17 / 41$ & $0.001^{b}$ \\
\hline EDSS & & 4 & & 2.3 & 5.5 & 3.0 & 5.0 & $0.001^{a}$ \\
\hline median (range) & & $(1.5-7.0)$ & & $(1.5-3.5)$ & $(3.5-7.0)$ & $(2.0-5.0)$ & $(2.5-7.0)$ & \\
\hline MSSS & & 5.3 & & 6.3 & 5.3 & 5.4 & 4.9 & 0.122 \\
\hline median (range) & & $(1.2-9.5)$ & & $(2.1-8.6)$ & $(3.7-9.1)$ & $(1.5-8.6)$ & $(1.6-9.4)$ & \\
\hline Disease duration (y) & & 11 & & 1 & 13 & 7 & 16 & $0.001^{a}$ \\
\hline median (range) & & $(0-35)$ & & $(0-11)$ & $(4-24)$ & $(1-29)$ & $(3-35)$ & \\
\hline Onset age (y) & & 28.0 & & 29.3 & 35.0 & 27.6 & 27.0 & $0.036^{c}$ \\
\hline median (range) & & $(13.0-57.0)$ & & $(18.5-41.0)$ & $(18-57)$ & $(15-46)$ & $(13-44)$ & \\
\hline
\end{tabular}

${ }^{\mathrm{a} A g e, ~ E D S S}$, and disease duration were significantly higher in PPMS and SPMS patients than in CIS and RRMS patients.

${ }^{\mathrm{b}}$ Male sex was significantly prevalent in PP patients.

'Onset age was significantly higher in PPMS patients than in RR and SP patients.

Abbreviations: CIS: clinically isolated syndrome; HCs: healthy controls; MS: multiple sclerosis; PPMS: primary progressive multiple sclerosis; RRMS: relapsing-remittent multiple sclerosis; SPMS: secondary progressive multiple sclerosis. 


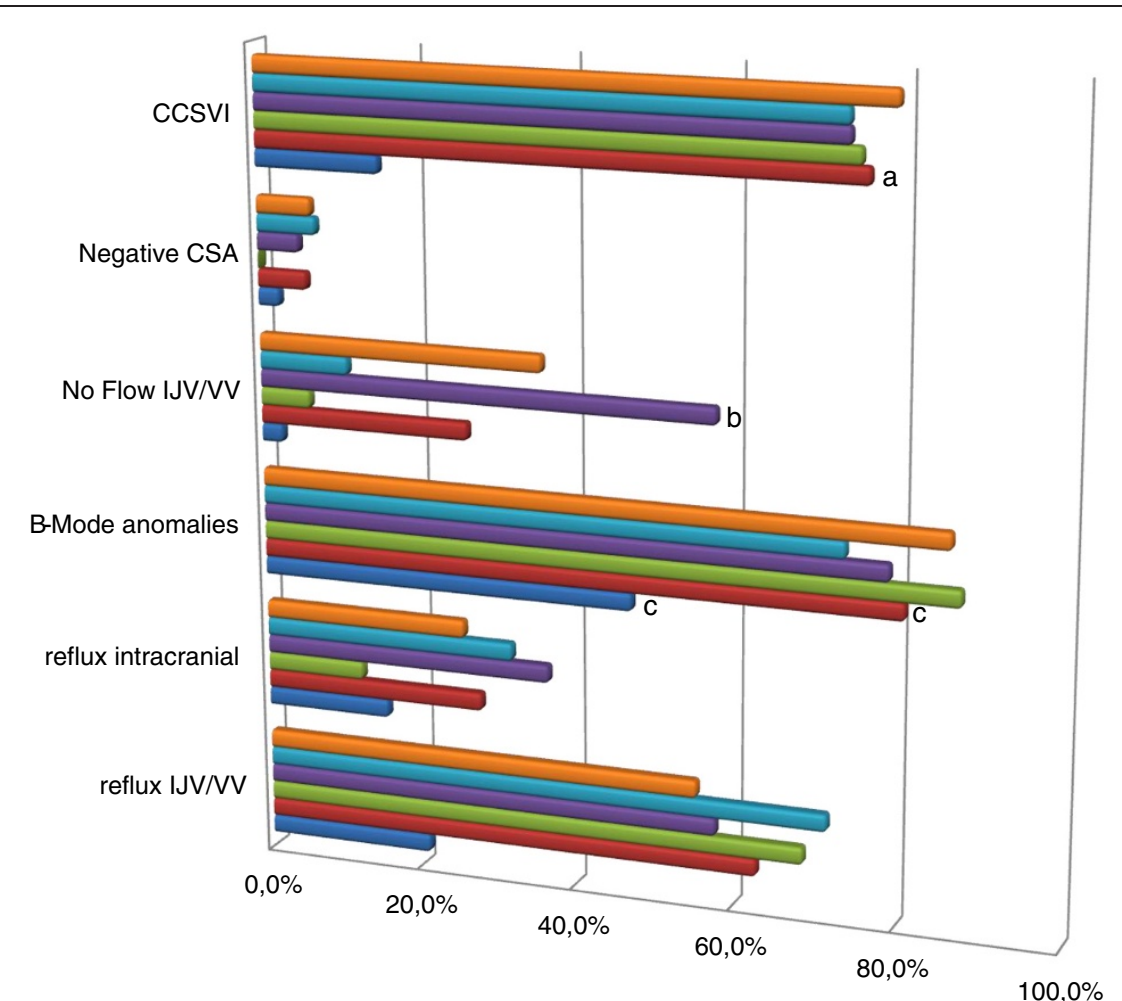

\begin{tabular}{|l|c|c|c|c|c|c|}
\cline { 2 - 6 } \multicolumn{1}{c|}{} & reflux IJV/VV & $\begin{array}{c}\text { reflux } \\
\text { intracranial }\end{array}$ & $\begin{array}{c}\text { B-Mode } \\
\text { anomalies }\end{array}$ & No Flow IJV/VV & Negative CSA & CCSVI \\
\hline$\square$ spMS & $55,5 \%$ & $25,9 \%$ & $86,2 \%$ & $36,2 \%$ & $6,9 \%$ & $79,3 \%$ \\
\hline$\square$ rrMS & $71,7 \%$ & $32,1 \%$ & $73,6 \%$ & $11,3 \%$ & $7,5 \%$ & $73,6 \%$ \\
\hline$\square$ ppMS & $57,9 \%$ & $36,8 \%$ & $78,9 \%$ & $57,9 \%$ & $5,3 \%$ & $73,7 \%$ \\
\hline$\square \mathrm{CIS}$ & $68,8 \%$ & $12,5 \%$ & $87,5 \%$ & $6,3 \%$ & $0,1 \%$ & $75,0 \%$ \\
\hline$\square \mathrm{MS}$ tot. & $63,0 \%$ & $28,1 \%$ & $80,8 \%$ & $26,7 \%$ & $6,2 \%$ & $76,0 \%$ \\
\hline$\square \mathrm{HC}$ & $21,1 \%$ & $15,8 \%$ & $47,4 \%$ & $2,6 \%$ & $2,6 \%$ & $15,8 \%$ \\
\hline
\end{tabular}

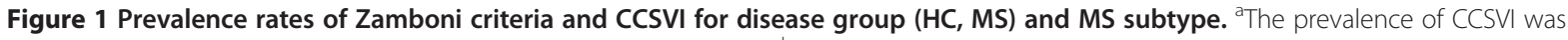

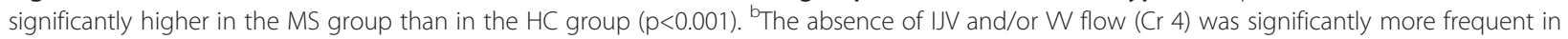
the PPMS form of disease compared with the RRMS form $(p<0.005)$. 'B-mode anomalies were the most frequently detected anomalies in MS patients and HCs. Abbreviations: CIS: clinically isolated syndrome; CCSVI: chronic cerebrospinal venous insufficiency; HCs: healthy controls; MS: multiple sclerosis; PPMS: primary progressive multiple sclerosis; RRMS: relapsing-remittent multiple sclerosis; SPMS: secondary progressive multiple sclerosis; Cr: Echo-Doppler criterion.

\section{Discussion}

We assessed the presence of CCSVI in MS patients and HCs, with a high interrater concordance $(85.4 \%$ and $92.7 \%$, respectively). Our study showed a higher prevalence of CCSVI in MS patients and a relatively higher specificity of CCSVI for MS, with a high OR for the diagnosis of MS vs HCs. The presence of CCSVI was strongly related to age in the whole sample and in MS patients, without any other clinical correlation. Single venous criteria were also related to age, and the absence of detectable flow in the IJV and/or VV (criterion 4) was more prevalent in PPMS patients compared with the other forms of MS, and was also related to age.

Our results are consistent with other studies that reported an increased prevalence of CCSVI in MS, despite our finding that sensitivity and specificity were lower than previously reported values $[4,9,10]$. Our finding of a higher prevalence of criterion 4 in PPMS (58\%) is also consistent with other studies that showed significantly more MR venography abnormalities in PP patients [24], as well as a longer cerebral circulation time in patients with progressive MS compared with nonprogressive MS [25,26], suggesting a role of hypoperfusion in neurodegeneration.

We did not find any relation of CCSVI with any clinical or demographic parameters, apart from age. These results do not indicate that CCSVI has a primary causative role in MS, as recently suggested in a recent review [27]. Moreover, a strong correlation between CCSVI and age, especially in MS patients, does not favour the hypothesis of the congenital nature of neck vascular alterations. The absence 
Table 2 Sensitivity, specificity, and odds ratios of single colour Doppler sonography criteria in MS patients and HCs

\begin{tabular}{|c|c|c|c|}
\hline & Sensitivity, \% (95\% Cl) & Specificity, \% (95\% Cl) & Odds ratio, $(95 \% \mathrm{Cl})$ \\
\hline \multirow[t]{2}{*}{ Reflux of the IJV and/or W $(\mathrm{Cr}$ 1) } & 63,0 & 78,9 & 6,4 \\
\hline & $(55,0-70,5)$ & $(64,2-89,5)$ & $(2,7-14,9)$ \\
\hline \multirow[t]{2}{*}{ Intracranial reflux (Cr 2) } & 28,1 & 84,2 & 2,1 \\
\hline & $(21,3-35,8)$ & $(70,3-93,1)$ & $(0,8-5,4)$ \\
\hline \multirow[t]{2}{*}{ IJV stenosis B-mode anomalies ( $\mathrm{Cr} 3$ ) } & 80,8 & 52,6 & 4,7 \\
\hline & $(73,9-86,6)$ & $(37,1-67,8)$ & $(2,2-10,0)$ \\
\hline \multirow[t]{2}{*}{ No flow in the IJV and/or W $(\mathrm{Cr} 4)$} & 26,7 & 97,4 & 13,5 \\
\hline & $(20,0-34,3)$ & $(88,4-99,6)$ & $(1,8-101,6)$ \\
\hline \multirow[t]{2}{*}{ Negative CSA ( $\mathrm{Cr} 5)$} & 6,2 & 97,4 & 2,4 \\
\hline & $(3,1-10,9)$ & $(88,4-99,6)$ & $(0,3-19,8)$ \\
\hline \multirow[t]{2}{*}{ CCSVI } & 76,0 & 84,2 & 16,9 \\
\hline & $(68,6-82,4)$ & $(70,3-93,1)$ & $(6,5-43,8)$ \\
\hline
\end{tabular}

An odds ratio greater than 1.0 indicates an increased likelihood of a diagnosis of CCSVI in MS patients versus controls. Abbreviations: MS: multiple sclerosis; HCs: healthy controls; CCSVI: chronic cerebrospinal venous insufficiency; Cr: criterion; IJV: internal jugular vein; VV: vertebral vein; CSA: cross sectional area; $\mathrm{Cl}$ : confidence interval.

of such a correlation in HCs in our study might be because of the small number of subjects in this group (power=30\%). Haemodynamic alterations in neck veins, such as jugular reflux in elderly people, have been described, and interestingly, they are associated with infratentorial white matter hyperintensity [28].

With regard to our finding of the correlation of criterion 4 with progressive forms of disease, the absence of flow in the IJV and/or VV could be related to factors that determine extrinsic compression of neck veins. The IJV passes below the sternocleidomastoid muscle to join the subclavian vein. Therefore, contraction of neck muscles or rotation of the head may result in a compression of these veins with a significant reduction in blood flow, even approaching complete occlusion [29]. The relation between vein biomechanics, surrounding muscle tone and trophism, and the fascial envelope has been demonstrated in the leg [30,31], but not in the IJVs. Moreover, in our study, the examiner paid attention to the inclination of the patient's head and used an appropriate neck support to avoid hyperextension or rotation of the head. However, it is not possible to exclude with certainty the influence of neck muscles on venous haemodynamics, especially in patients with higher neurological disability. In the current study, progressive patients were older than nonprogressive patients, and criterion 4, similar to criteria 1 and 3 , was related to age, confirming that this appears to strongly influence venous hemodynamic alterations in MS. Indeed, PP and SP patients who showed a similar mean age had a comparable prevalence of criterion $4(\mathrm{p}=0.053)$.

However, it remains unknown if CCSVI is a causal factor or a consequence of MS. Our findings suggest that CCSVI may be a secondary effect rather than a cause of MS, and they indicate that in MS patients, venous valves become less continent with ageing, in association with morphological and functional changes. Many reports have shown the presence of ageing-related increased fibrosis and thickening of the valve leaflets and vein wall [32-36], and decreased compliance of the vein wall [37-39]. Changes in compliance in the vein wall affect venous blood flow, and thickened, stiff leaflets disrupt normal blood flow during the valvular cycle. Several studies have reported hypoperfusion of brain parenchyma in patients with MS, advancing with disease progression [40-43], and it is possible that neck venous anomalies develop secondarily to reduced perfusion. The combination of chronic hypoxia or intermittent hypoxia and haemodynamic factors creates conditions that convert the endothelium of veins to a proinflammatory and procoagulant phenotype [37], which is characterized by the production of plasminogen activator inhibitor-1, release of von Willebrand factor, exposure of P-selectin, secretion of the vasoconstrictor endothelin-1, and production and release of reactive oxygen species. Moreover, hemodynamic forces are important modulators of vascular structure. In rabbits, progressive intimal thickening occurs when shear stress is reduced to subnormal levels with preserved endothelium [44]. There is no evidence of inflammatory infiltrates in neck vein walls in MS [26]. In MS patients, vein walls appear to undergo remodelling of collagen fibres, altering the collagen I/III proportion [26]. The presence of collagen alterations might be due to age-related changes in the vein wall and valves, which have been described in renal veins [33]. In our study, changes corresponding to those found in normal ageing of veins were found in MS patients, and interestingly, this phenomenon was more evident in progressive MS forms where changes in the vein wall and valves caused the most serious hemodynamic consequences. 
An interesting hypothesis was recently raised in an article suggesting that a neurological process could play a role in CCSVI progression through vasoactive substances (e.g., endothelin-1) or proinflammatory agents, which could act on previously susceptible segments of blood vessels (i.e., malformed vein valves) [45]. This hypothesis would explain the higher prevalence of CCSVI in MS patients and its relation to patients' age. The same article also suggested a parallel between venous and aortic valves stenosis, which are different entities, but probably share common features. MS patient live in a state of venous stasis that can be attributed to excessive ageing of veins and venules secondary to three elements: 1) chronic inflammation that induces changes in structure and function of the vein wall (in particular, the endothelium); 2) MS-associated alteration in blood flow; and 3) a shift to a hypercoagulable state [46,47]. These changes are located in the trunk wall and the lesions are non-homogeneous and dispersed. The development of new and modern biomedical imaging techniques would be useful for investigating venous valvular pathophysiology in animal models and human subjects.

\section{Conclusions}

In conclusion, our results suggest a higher prevalence of CCSVI in MS patients, which increases with age, possibly more precociously in MS patients than in healthy people, but without any correlation with disability and disease progression. Normal "ageing" of neck veins should be investigated and it should be verified whether reduced cerebral blood flow and cerebral venular chronic inflammatory exposure, both evident in MS, induce venous brain outflow alterations. We believe that to determine the relationship between CCSVI and MS, a more sophisticated assessment is necessary, especially at the beginning of disease and with a longitudinal prospective.

In particular, more refined and quantitative measures, such as blood flow velocity, volume flow, and cerebral circulation times, could be more reliable in assessing the physiological relevance of venous outflow abnormalities.

\section{Abbreviations}

MS: Multiple sclerosis; CCSVI: Chronic cerebrospinal venous insufficiency; ECD: Echo-colour Doppler; HC: Healthy control; RR: Relapsing remittent; SP: Secondary progressive; PP: Primary progressive; CIS: Clinically isolated syndrome; MRV: Magnetic resonance venography; EDSS: Expanded disability status scale; IJV: Internal jugular vein; W: Vertebral vein.

\section{Competing interests}

The authors declare that they have no competing interests.

\section{Authors' contributions}

RoL was involved in study concept/study design, data acquisition, data analysis/interpretation, manuscript drafting, literature research, and clinical studies. MM was involved in the study concept/study design, data acquisition, data analysis/interpretation, manuscript drafting, and literature research. RaL performed statistical analysis, drafted the manuscript, and performed literature research. ODD performed data acquisition and literature research. ES performed clinical studies and data acquisition. VM performed data acquisition and literature research. GV and LA performed literature research. GD performed data acquisition and edited the manuscript. $A B$ participated in the study concept/study design. VBM was involved in study concept/study design, data acquisition, data analysis/interpretation, manuscript drafting, literature research, and clinical studies. All authors read and approved the final manuscript.

\section{Acknowledgements}

This work was supported in part by a research grant from the Italian Ministry for Education University and Research in the framework of PRIN (2010XE5L2R_004).

\section{Author details}

${ }^{1}$ Department of Neurological Sciences, Federico II University School of Medicine, Naples, Italy. ${ }^{2}$ Institute of Biostructure and Bioimaging, National Research Council of Italy (CNR), Naples, Italy. ${ }^{3}$ IRCCS, SDN Foundation, Institute of Diagnostic and Nuclear development, Naples, Italy. ${ }^{4}$ Department of Biomorphological and Functional Science, Federico II University School of Medicine, Naples, Italy. ${ }^{5}$ Hermitage Capodimonte IDC, Naples, Italy.

Received: 3 August 2012 Accepted: 4 February 2013

Published: 13 February 2013

\section{References}

1. Frohman EM, Racke MK, Raine CS: Multiple sclerosis-the plaque and its pathogenesis. N Engl J Med 2006, 354(9):942-955.

2. Lassmann H: Hypoxia-like tissue injury as a component of multiple sclerosis lesions. J Neurol Sci 2003, 206(2):187-191.

3. Law M, Saindane AM, Ge Y, et al: Microvascular abnormality in relapsingremitting multiple sclerosis: perfusion MR imaging findings in normalappearing white matter. Radiology 2004, 231(3):645-652.

4. Zamboni P, Galeotti R, Menegatti E, et al: Chronic cerebrospinal venous insufficiency in patients with multiple sclerosis. J Neurol Neurosurg Psychiatry 2009, 80(4):392-399.

5. Lugli M, Morelli M, Guerzoni S, et al: The hypothesis of patho-physiological correlation between chronic cerebrospinal venous insufficiency and multiple sclerosis: rationale of treatment. Phlebology 2012, 27(Suppl 1):178-186.

6. Simka M, Latacz P, Ludyga T, et al: Prevalence of extracranial venous abnormalities: results from a sample of 586 multiple sclerosis patients. Funct Neurol 2011, 26(4):197-203.

7. Simka M, Ludyga T, Latacz $P$, et al: Diagnostic accuracy of current sonographic criteria for the detection of outflow abnormalities in the internal jugular veins. Phlebology 2012, doi:10.1258/phleb.2012.011125.

8. Yamout B, Herlopian A, Issa Z, et al: Extracranial venous stenosis is an unlikely cause of multiple sclerosis. Mult Scler 2010, 16(11):1341-1348.

9. Hojnacki D, Zamboni P, Lopez-Soriano A, et al: Use of neck magnetic resonance venography, Doppler sonography and selective venography for diagnosis of chronic cerebrospinal venous insufficiency: a pilot study in multiple sclerosis patients and healthy controls. Int Angiol 2010, 29(2):127-139.

10. Zamboni P, Galeotti R, Menegatti E, et al: A prospective open-label study of endovascular treatment of chronic cerebrospinal venous insufficiency. J Vasc Surg 2009, 50(6):1348-1358. e1341-1343.

11. Baracchini $C$, Perini $P$, Calabrese $M$, et al: No evidence of chronic cerebrospinal venous insufficiency at multiple sclerosis onset. Ann Neurol 2011, 69(1):90-99.

12. Zamboni $P$, Menegatti $E_{1}$ Galeotti $R$, et al: The value of cerebral Doppler venous haemodynamics in the assessment of multiple sclerosis. J Neurol Sci 2009, 282(1-2):21-27.

13. Tsivgoulis G, Mantatzis M, Bogiatzi C, et al: Extracranial venous hemodynamics in multiple sclerosis: a case-control study. Neurology 2011, 77(13):1241-1245.

14. Zivadinov R, Marr K, Cutter G, et al: Prevalence, sensitivity, and specificity of chronic cerebrospinal venous insufficiency in MS. Neurology 2011, 77(2):138-144.

15. Garaci FG, Marziali S, Meschini A, et al: Brain hemodynamic changes associated with chronic cerebrospinal venous insufficiency are not specific to multiple sclerosis and do not increase its severity. Radiology 2012, 265(1):233-239.

16. Zamboni $P$, Menegatti $E$, Weinstock-Guttman B, et al: Hypoperfusion of brain parenchyma is associated with the severity of chronic 
cerebrospinal venous insufficiency in patients with multiple sclerosis: a cross-sectional preliminary report. BMC Med 2011, 9:22.

17. Baracchini $C$, Perini $P$, Causin F, et al: Progressive multiple sclerosis is not associated with chronic cerebrospinal venous insufficiency. Neurology 2011, 77(9):844-850.

18. Bastianello S, Romani A, Viselner G, et al: Chronic cerebrospinal venous insufficiency in multiple sclerosis: clinical correlates from a multicentre study. BMC Neurol 2011, 11:132

19. Centonze D, Floris R, Stefanini M, et al: Proposed chronic cerebrospinal venous insufficiency criteria do not predict multiple sclerosis risk or severity. Ann Neurol 2011, 70(1):51-58.

20. McDonald WI, Compston A, Edan G, et al: Recommended diagnostic criteria for multiple sclerosis: guidelines from the International Panel on the diagnosis of multiple sclerosis. Ann Neurol 2001, 50(1):121-127.

21. Lublin FD, Reingold SC: Defining the clinical course of multiple sclerosis: results of an international survey. National Multiple Sclerosis Society (USA) Advisory Committee on Clinical Trials of New Agents in Multiple Sclerosis. Neurology 1996, 46(4):907-911.

22. Kurtzke JF: Rating neurologic impairment in multiple sclerosis: an expanded disability status scale (EDSS). Neurology 1983, 33(11):1444-1452.

23. Roxburgh RH, Seaman SR, Masterman T, et al: Multiple Sclerosis Severity Score: using disability and disease duration to rate disease severity. Neurology 2005, 64(7):1144-1151.

24. Dolic K, Marr K, Valnarov V, et al: Intra- and extraluminal structural and functional venous anomalies in multiple sclerosis, as evidenced by 2 noninvasive imaging techniques. AJNR Am J Neuroradiol 2012, 33(1):16-23.

25. Mancini M, Morra VB, Di Donato O, et al: Multiple sclerosis: cerebral circulation time. Radiology 2012, 262(3):947-955.

26. Coen M, Menegatti E, Salvi F, et al: Altered collagen expression in jugular veins in multiple sclerosis. Cardiovasc Pathol 2013, 22(1):33-38.

27. Lee $A B$, Laredo J, Neville R: Embryological background of truncular venous malformation in the extracranial venous pathways as the cause of chronic cerebro spinal venous insufficiency. Int Angiol 2010, 29(2):95-108.

28. Chung $\mathrm{CP}$, Wang $\mathrm{PN}, \mathrm{Wu} \mathrm{YH}$, et al: More severe white matter changes in the elderly with jugular venous reflux. Ann Neurol 2011, 69(3):553-559.

29. Muhammad JK, Pugh ND, Boden $L$, et al: The effect of head rotation on the diameter of the internal jugular vein: implications for free tissue transfer. J Craniomaxillofac Surg 2001, 29(4):214-218.

30. Buckey JC, Lane LD, Plath $G$, et al: Effects of head-down tilt for 10 days on the compliance of the leg. Acta Physiol Scand Suppl 1992, 604:53-59.

31. Buckey JC, Peshock RM, Blomqvist CG: Deep venous contribution to hydrostatic blood volume change in the human leg. Am J Cardiol 1988, 62(7):449-453

32. Gottlob MMR: Pathologic venous valves. In Venous Valves: Morphology, Function Radiology, Surgery. Edited by Gottlob RM R. New York: Springer; 1986:82-92.

33. Chopard RP, Miranda Neto MH, Biazotto W, et al: Age-related changes in the human renal veins and their valves. Ital J Anat Embryol 1994, 99(2):91-101.

34. Saphir O, Lev M: The venous valve in the aged. Am Heart J 1952, 44(6):843-850

35. Schina MJ Jr, Neumyer MM, Healy DA, et al: Influence of age on venous physiologic parameters. J Vasc Surg 1993, 18(5):749-752.

36. van Langevelde K, Sramek A, Rosendaal FR: The effect of aging on venous valves. Arterioscler Thromb Vasc Biol 2010, 30(10):2075-2080.

37. Lurie F, Kistner RL, Eklof B, et al: Mechanism of venous valve closure and role of the valve in circulation: a new concept. J Vasc Surg 2003, 38(5):955-961.

38. Olsen $\mathrm{H}$, Lanne T: Reduced venous compliance in lower limbs of aging humans and its importance for capacitance function. Am J Physiol 1998, 275(3 Pt 2):H878-886.

39. Young CN, Stillabower ME, DiSabatino A, et al: Venous smooth muscle tone and responsiveness in older adults. J Appl Physiol 2006, 101(5):1362-1367.

40. De Keyser J, Steen C, Mostert JP, et al: Hypoperfusion of the cerebral white matter in multiple sclerosis: possible mechanisms and pathophysiological significance. J Cereb Blood Flow Metab 2008, 28(10):1645-1651.
41. Inglese M, Adhya S, Johnson $\mathrm{G}$, et al: Perfusion magnetic resonance imaging correlates of neuropsychological impairment in multiple sclerosis. J Cereb Blood Flow Metab 2008, 28(1):164-171.

42. Varga AW, Johnson G, Babb JS, et al: White matter hemodynamic abnormalities precede sub-cortical gray matter changes in multiple sclerosis. J Neurol Sci 2009, 282(1-2):28-33.

43. Wuerfel J, Paul F, Zipp F: Cerebral blood perfusion changes in multiple sclerosis. J Neurol Sci 2007, 259(1-2):16-20.

44. Zhuang YJ, Singh TM, Zarins $C K$, et al: Sequential increases and decreases in blood flow stimulates progressive intimal thickening. Eur J Vasc Endovasc Surg 1998, 16(4):301-310.

45. Simka M, Ludyga T, Kazibudzki M, et al: Multiple sclerosis, an unlikely cause of chronic cerebrospinal venous insufficiency: retrospective analysis of catheter venography. J R Soc Med Sh Rep 2012, 3(8):6.

46. Aksungar FB, Topkaya AE, Yildiz Z, et al: Coagulation status and biochemical and inflammatory markers in multiple sclerosis. J Clin Neurosci 2008, 15(4):393-397.

47. Vandenberghe $N$, Debouverie $M$, Anxionnat $R$, et al: Cerebral venous thrombosis in four patients with multiple sclerosis. Eur I Neurol 2003, 10(1):63-66.

doi:10.1186/1471-2377-13-20

Cite this article as: Lanzillo et al:: Chronic cerebrospinal venous

insufficiency in multiple sclerosis: a highly prevalent

age-dependent phenomenon. BMC Neurology 2013 13:20

\section{Submit your next manuscript to BioMed Central and take full advantage of:}

- Convenient online submission

- Thorough peer review

- No space constraints or color figure charges

- Immediate publication on acceptance

- Inclusion in PubMed, CAS, Scopus and Google Scholar

- Research which is freely available for redistribution 